\title{
Activities of E6 Protein of Human Papillomavirus 16 Asian Variant on miR-21 Up-regulation and Expression of Human Immune Response Genes
}

\author{
Peechanika Chopjitt ${ }^{1,3}$, Chamsai Pientong ${ }^{1,3}$, Sureewan Bumrungthai ${ }^{1,3}$, Bunkerd \\ Kongyingyoes $^{2}$, Tipaya Ekalaksananan ${ }^{1,3 *}$
}

\begin{abstract}
Background: Variants of human papillomavirus (HPV) show more oncogenicity than do prototypes. The HPV16 Asian variant (HPV16As) plays a major role in cervical cancer of Asian populations. Some amino acid changes in the E6 protein of HPV16 variants affect E6 functions such as p53 interaction and host immune surveillance. This study aimed to investigate activities of HPV16As E6 protein on modulation of expression of miRNA-21 as well as interferon regulatory factors (IRFs) 1,3,7 and c-fos. Materials and Methods: Vectors expressing E6 protein of HPV16As (E6D25E) or HPV16 prototype (E6Pro) were constructed and transfected into C33A cells. HCK1T cells expressing E6D25E or E6Pro were established by transducing retrovirus-containing E6D25E or 16E6Pro. The E6AP-binding activity of $\mathrm{E} 6$ and proliferation of the transfected $\mathrm{C33A}$ cells were determined. MiR-21 and mRNA of interesting genes were detected in the transfected C33A cells and/or the HCK1T cells, with or without treatment by culture medium from HeLa cells (HeLa-CM). Results: E6D25E showed binding activity with E6AP similar to that of E6Pro. Interestingly, E6D25E showed a higher activity of miR-21 induction than did E6Pro in C33A cells expressing E6 protein. This result was similar to the HCK1T cells expressing E6 protein, with HeLa-CM treatment. The miR-21 up-regulation significantly corresponded to its target expression. Different levels of expression of IRFs were also observed in the HCK1T cells expressing E6 protein. Interestingly, when treated with HeLa-CM,IRFs 1,3 and 7 as well as c-fos were significantly suppressed in the HCK1T cells expressing E6D25E, whereas those in the HCK1T cells expressing E6Pro were induced. A similar situation was seen for IFN- $\alpha$ and IFN- $\beta$. Conclusions: E6D25E of the HPV16As variant differed from the E6 prototype in its activities on epigenetic modulation and immune surveillance and this might be a key factor for the important role of this variant in cervical cancer progression.
\end{abstract}

Keywords: HPV16 Asian variant - E6D25E - miR-21, IRFs - c-fos

Asian Pac J Cancer Prev, 16 (9), 3961-3968

\section{Introduction}

Cervical cancer is the third most common malignancy affecting women worldwide. Infection with human papillomavirus (HPV), especially HPV type 16 (HPV16), is recognized as the major cause of cervical cancer in high risk groups (HR-HPV) (Walboomers et al., 1999). HPV 16 was found in $83.2 \%$ of Thai women in one study (Suthipintawong et al., 2011). HPV16 E6 and E7 proteins are characterized as oncoproteins. The E6 protein binds E6-associated protein (E6AP) then forms a complex with the p53 tumor suppressor protein to degrade p53 through the ubiquitin pathway leading to loss of cell cycle control and anti-apoptosis. The E7 protein binds to the retinoblastoma family proteins, resulting in loss of the checkpoint control and uncontrolled cell proliferation. (Zhang and Tang, 2012)
HPV16 has been extensively sequenced to characterize subtypes and variants in a phylogenetic framework. According to geographic location and ethnic group, HPV16 variants have been classified into 4 lineages; 1 ) European-Asian (EAs) including the sublineage European (E, the prototype of HPV16) and Asian (As), 2) African 1 (AFR1), 3) African 2 (AFR2) and 4) North American/ Asian-American (NA/AA) including the sublineage North American, Asian-American 1 and Asian-American 2 (Yamada et al., 1997; Cornet et al., 2012). Epidemiological studies show an association between cervical cancer and HPV16 variants and report that HPV16 E6 variants may be more oncogenic than the prototype and thus carry a higher risk for development of invasive cervical disease (Xi et al., 2007; Schiffman et al., 2010; Gheit et al., 2011; Mosmann et al., 2015). Many studies have shown high prevalence of the HPV16 Asian variant or HPV16As

${ }^{I}$ Department of Microbiology, ${ }^{2}$ Department of Pharmacology, Faculty of Medicine, ${ }^{3} \mathrm{HPV} \&$ EBV and Carcinogenesis Research Group, Khon Kaen University, Khon Kaen, Thailand *For correspondence: tipeka@kku.ac.th 
(member of EA lineages) in invasive cervical cancer and an association with cervical cancer progression in Asian populations (Kang et al., 2005; Choi et al., 2007; Chopjitt et al., 2009; Chang et al., 2013; Pientong et al., 2013). The E6 gene in this variant encodes glutamic acid (E) rather than aspartic acid (D) at position 25 (D25E). Modification of E6 protein activity has an important effect on carcinogenesis. Several amino acid changes in HPV16 E6 have been identified in positions crucial for p53 interaction and in positions likely to be important for host immune surveillance (Zehbe et al., 1998).

MicroRNAs (miRNA or miR-) are small non-coding RNA molecules of 19-25 nucleotides in length. MiRNAs have regulatory functions involved in several processes such as cell differentiation, apoptosis and cell-proliferation (Kavitha et al., 2014). HR-HPV E6 and E7 proteins expressed in human foreskin keratinocytes (HFKs) also share the ability to regulate a subset of cellular miRNAs (Yablonska et al., 2013). Numerous miRNAs have been identified that are associated with cervical carcinogenesis. For example, down-regulation of miR-218 leads to promotion of cancer cell migration and invasion ( $\mathrm{Li}$ et al., 2010) and up-regulation of miR-205 enhances cell proliferation and migration (Xie et al., 2012). Multiple studies have noted that miR-21 plays a significant role in cancer biology, promotes cell proliferation, migration and invasion, prevents apoptosis in diverse cancers and cancer cell lines. Moreover, miR-21 has been reported as a novel diagnostic and prognostic marker for epithelial ovarian cancer (Xu et al., 2013).

Innate immunity is the first line defense against viral infection (Sasagawa et al., 2012). IFN type I, IFN- $\alpha$ and IFN- $\beta$ production are activated early after viral infection. IFN- $\beta$ is produced by three different ways of transcriptional interaction, including c-jun/ATF2 (AP1), interferon regulatory factor (IRF) and NFxB. Induction of IFN- $\alpha$ is activated by IFRs, and distinct DNA binding characteristics and patterns of activation provide the differential expression of the IFN- $\alpha$ gene family (Smith et al., 2001). IRFs play a role as upstream molecules regulating IFN type I expression. In HPV-infected cells, E6 and E7 oncoproteins have been implicated in IFN production (Ronco et al., 1998; Barnard and McMillan, 1999; Park et al., 2000; Um et al., 2002).

To understand the role of HPV16As in cervical cancer development, this study compared the activity of E6 protein of HPV16As (E6As or E6D25E) with E6 from the HPV16 prototype (E6Pro) on production of miR-21, IRF 1,3 and 7 and c-fos in C33A and HCK1T cells.

\section{Materials and Methods}

\section{Cell lines}

C33A, an HPV-negative cervical cancer cell line and HeLa HPV18-positive cervical cancer cell line were cultured in DMEM medium (Gibco®, by Life Technologies, Grand Island, NY, USA) supplemented with $10 \%$ fetal bovine serum (FBS, Gibco ${ }^{\circledR}$, by Life Technologies, Grand Island, NY, USA) and antibiotics at $37^{\circ} \mathrm{C}$ in a humidified atmosphere with $5 \% \mathrm{CO}_{2}$. HCK1T, normal diploid human cervical keratinocytes, with the capacity for normal differentiation established by transducing the catalytic subunit of human telomerase reverse transcriptase (hTERT) (Narisawa-Saito et al., 2008), were cultured in serum-free medium (KSFM, Gibco®, by Life Technologies, Grand Island, NY, USA) at $37^{\circ} \mathrm{C}$ in a humidified atmosphere with $5 \% \mathrm{CO}_{2}$.

\section{Plasmids}

Plasmids used in this study consisted of an E6AP expression plasmid (pcDNA3-E6AP, kindly provided by Jon M. Huibregtse (Huibregtse et al., 1993)), E6 protein expression plasmids (pcDNA3.2-16E6Pro and pcDNA3.2-16E6D25E) and retrovirus expression plasmids (pCLXSN, pCLXSN-16E6Pro and pCLXSN16E6D25E).

The plasmid pcDNA3.2-16E6Pro was constructed by sub-cloning the E6 gene from a reference plasmid HPV 16 prototype (kindly provided by Ethel-Michele de Villiers) into pcDNA3.2/V5/GW/D-TOPO® vector using pcDNA ${ }^{\mathrm{TM}} 3.2 / \mathrm{GW} / \mathrm{D}-\mathrm{TOPO}{ }^{\circledR}$ Expression Kit (InvitrogenTM Carlsbad, CA, USA) according to the manufacturer's instruction. The plasmid pcDNA3.216E6D25E was constructed using the E6 gene amplified from HPV16As in a cervical cancer sample, identified as previously described by us (Chopjitt et al., 2009). Construction of the retrovirus expression vector pCLXSN16E6Pro was described previously (Kiyono et al., 1997). Vector pCLXSN-16E6D25E was similarly constructed using the Gateway system (Invitrogen, Carlsbad, CA). Briefly, entry vectors containing E6D25E were constructed from pENTR201-16E6 using site-directed mutagenesis (QuikChange Site-Directed Mutagenesis Kit, Stratagene, La Jolla, CA, USA). These entry vectors were recombined into the destination vector, pDEST-CLXSN using the LR reaction (Invitrogen, Carlsbad, CA, USA) to generate pCLXSN-16E6D25E.

\section{Determination of E6 protein and E6AP interaction}

C33A cells were co-transfected with pcDNA3.216E6Pro and pcDNA3-E6AP, or with pcDNA3.2$16 \mathrm{E} 6 \mathrm{D} 25 \mathrm{E}$ and pcDNA3-E6AP and total protein was extracted using lysis buffer. Lysate was precleared with 20 $\mu l$ Protein G-PLUS-Agarose (Santa Cruz Biotechnology, Santa Cruz, CA, USA) and incubated at $4^{\circ} \mathrm{C}$ for $1 \mathrm{~h}$ with rocking, then centrifuged at 2,000 rpm for $5 \mathrm{~min}$ at $4^{\circ} \mathrm{C}$. The supernatant was collected and proteins were quantified using the Bio-Rad Protein assay (Bio-Rad, Hercules, CA, USA). Two $\mu \mathrm{g}$ of anti-HPV16/18 E6 antibody (Abcam, Cambridge, UK) was added to the protein lysate (500 $\mu \mathrm{g}$ of total protein) and incubated for $1 \mathrm{~h}$ at $4^{\circ} \mathrm{C}$. Twenty $\mu \mathrm{l}$ of Protein G- PLUS-Agarose was added and incubated at $4^{\circ} \mathrm{C}$ on a rotating device overnight. The protein complex was collected by centrifugation at 2,500 rpm for $5 \mathrm{~min}$ at $4^{\circ} \mathrm{C}$ and the pellet washed twice with RIPA buffer and twice with PBS. The pellet was resuspended in $30 \mu \mathrm{l}$ of sample buffer. To monitor the E6/E6AP interaction, samples were boiled for $5 \mathrm{~min}$ and visualized using western blot assay. Proteins were quantified using the Bio-Rad Protein assay, resuspended in $50 \mu 1$ of sample buffer, separated by $10 \%$ SDS-PAGE, and then transferred onto a PVDF membrane. The membrane was incubated with anti-E6AP (Santa 
Cruz Biotechnology, Santa Cruz, CA, USA) overnight at $4 \mathrm{oC}$, and then incubated with HRP-conjugated antimouse secondary antibody (Santa Cruz Biotechnology, Santa Cruz, CA, USA) for $1 \mathrm{~h}$ at room temperature. The reaction was then visualized using a SuperSignal West Pico Chemiluminescent Substrate (Thermo scientific, IL, USA) with lmageQuant LAS 4000 mini (GE Healthcare, Piscataway, NY, USA).

\section{Cell cycle analysis}

Cell-cycle analysis was performed by flow cytometry using propidium iodide (PI) staining. In brief, C33A cells $\left(2 \times 10^{5}\right.$ cells/well in 6 -well plates) were transfected with the empty plasmid (pcDNA3.2/V5) or pcDNA3.216E6D25E or pcDNA3.2-16E6Pro. After $48 \mathrm{~h}$, the harvested cells were fixed in cold $70 \%$ ethanol in PBS at $4^{\circ} \mathrm{C}$ overnight then washed twice with PBS. The cells were centrifuged at 2,000 rpm for $15 \mathrm{~min}$ and washed twice with PBS. The cells were stained with $3 \mu \mathrm{M}$ PI (Invitrogen, Paisley, UK) containing $0.1 \mathrm{mg} / \mathrm{ml}$ RNase in PBS for 30 min at room temperature. DNA content in 10,000 cells was analyzed with FACS DivaTM software on a FACSTM II flow cytometer (Becton Dickinson, San Jose, CA, USA) by gating on an area-versus-width dot plot to exclude cell debris and aggregates.

\section{Production and titration of recombinant retroviruses}

Recombinant retroviruses containing HPV16 E6 genes and control retrovirus were produced in the $293 \mathrm{~T}$ cell line. Briefly, each of the retrovirus expression plasmids (pCLXSN-16E6D25E, pCLXSN-16E6Pro and pCLXSN) and packaging plasmids (pCL-GagPol and pEF6-env (10A1)) were co-transfected into 293T cells using TransIT-293 (Mirus Co., Madison, USA) according to the manufacturer's instructions. Recombinant retroviruses in culture media were harvested at 60 to $72 \mathrm{~h}$ post-transfection. Titers of the recombinant retroviruses were measured using a G418 resistant colony assay in HeLa cells. For this assay, $5 \times 10^{4} \mathrm{HeLa}$ cells/well in 12-well plates were infected with serial dilutions of the recombinant retroviruses in $8 \mu \mathrm{g} / \mathrm{ml}$ of polybrene (Sigma, St Louis, MO, USA). After 48 h, cells were fed with fresh medium containing $800 \mu \mathrm{g} / \mathrm{ml}$ of G418 (Gibco®, by Life Technologies, Grand Island, NY, USA). Cell colonies were stained with Giemsa's dye and counted after 2 weeks.

\section{Establishment of HCK1T cell lines stably expressing E6 proteins}

HCK1T cell lines stably expressing E6 proteins were established by transduction of retrovirus vectors containing E6Pro and E6D25E genes. One day before transduction, HCK1T cells were plated at a density of $2 \times 10^{5}$ cells/well in 6-well plates. The recombinant retroviruses containing E6 genes were inoculated at 0.5 multiplicity of infection (MOI=0.5) for $2 \mathrm{~h}$ in the presence of $4 \mu \mathrm{g} / \mathrm{ml}$ polybrene. The medium was then replaced with fresh KSFM. Two days post-infection, cells were fed with fresh medium supplemented with $50 \mu \mathrm{g} / \mathrm{ml}$ of G418, until mock-infected cells died completely. Cells were fed with fresh medium every 3 days until cells reached subconfluence. HCK1T cell control was established by transduction with control retrovirus (lacking the E6 gene).

\section{Treatment of HCK1T cell lines stably expressing E6 proteins}

Medium in all established HCK1T cell lines at passage 25 was replaced with culture medium from HeLa cells (HeLa-CM) and incubated for $48 \mathrm{~h}$, then the treated cells were harvested and total RNA was extracted and used for investigation. Complete medium (CM, DMEM supplemented with $10 \%$ FBS) was used in controls.

\section{Detection of $m R N A$ and $m i R-21$}

Total RNA was extracted with TRIzol reagent (Ambion, Life technologies, Carlsbad, CA, USA) and cDNA was synthesized using the Super Script ${ }^{\circledR}$ III FirstStrand Synthesis System according to manufacturer's instruction (Invitrogen, Life technologies, Carlsbad,

Table 1. Nucleotide Sequence of Specific Primers and their PCR Conditions

\begin{tabular}{|c|c|c|}
\hline Genes & Sequence (5'-3') & Conditions \\
\hline \multirow[t]{2}{*}{ E6 } & Forward; TTGAACCGAAACCGGTTAGT & $95^{\circ} \mathrm{C}$ for $5 \mathrm{~min} ; 40$ cycles, $95^{\circ} \mathrm{C}$ for $1 \mathrm{~min}, 55^{\circ} \mathrm{C}$ for \\
\hline & Reverse; GCATAAATCCCGAAAAGCAA & $30 \mathrm{~s}, 72^{\circ} \mathrm{C}$ for $1 \mathrm{~min}$, final extension, $72^{\circ} \mathrm{C}$ for $5 \mathrm{~min}$ \\
\hline \multirow[t]{2}{*}{ PDCD4 } & Forward; GATTAACTGTGCCAACCAGTCCAAAG & $98^{\circ} \mathrm{C}$ for $30 \mathrm{~s}, 98^{\circ} \mathrm{C}$ for $1 \mathrm{~s}, 65^{\circ} \mathrm{C}$ for $1 \mathrm{~s}$ \\
\hline & Reverse; CATCCАССТССТССАСАТСАТАСАС & Melt curve; $65-95^{\circ} \mathrm{C}$ for $2 \mathrm{sec} / \mathrm{step}$ \\
\hline \multirow[t]{2}{*}{ Actin } & Forward; ACCAACTGGGACGACATGGAGAAA & $98^{\circ} \mathrm{C}$ for $30 \mathrm{~s}, 98^{\circ} \mathrm{C}$ for $1 \mathrm{~s}, 65^{\circ} \mathrm{C}$ for $1 \mathrm{~s}$ \\
\hline & Reverse; TAGCACAGCCTGGATAGCAACGTA & Melt curve; $65-95^{\circ} \mathrm{C}$ for $2 \mathrm{sec} / \mathrm{step}$ \\
\hline \multirow[t]{2}{*}{ IRF 1} & Forward; TCTTAGCATCTCGGCTGGACTTC & $98^{\circ} \mathrm{C}$ for $30 \mathrm{~s}, 98^{\circ} \mathrm{C}$ for $1 \mathrm{~s}, 65^{\circ} \mathrm{C}$ for $1 \mathrm{~s}$ \\
\hline & Reverse; CGATACAAAGCAGGGGAAAAGG & Melt curve; $65-95^{\circ} \mathrm{C}$ for $2 \mathrm{sec} / \mathrm{step}$ \\
\hline \multirow[t]{2}{*}{ IRF 3} & Forward; CAGGGCCTTGGTAGAAATGG & $98^{\circ} \mathrm{C}$ for $30 \mathrm{~s}, 98^{\circ} \mathrm{C}$ for $1 \mathrm{~s}, 65^{\circ} \mathrm{C}$ for $1 \mathrm{~s}$ \\
\hline & Reverse; GGGTGGCTGTTGGAAATGTG & Melt curve; $65-95^{\circ} \mathrm{C}$ for $2 \mathrm{sec} / \mathrm{step}$ \\
\hline \multirow{2}{*}{ IRF 7} & Forward; GATGTCGTCATAGAGGCT GTTGG & $98^{\circ} \mathrm{C}$ for $30 \mathrm{~s}, 98^{\circ} \mathrm{C}$ for $1 \mathrm{~s}, 65^{\circ} \mathrm{C}$ for $1 \mathrm{~s}$ \\
\hline & Reverse; TGGTCCTGGTGAAGCTGGAA & Melt curve; $65-95^{\circ} \mathrm{C}$ for $2 \mathrm{sec} / \mathrm{step}$ \\
\hline \multirow[t]{2}{*}{ IFN- $\alpha$} & Forward; AGGAGGAGTTTGATGGCAACCAGT & $98^{\circ} \mathrm{C}$ for $30 \mathrm{~s}, 98^{\circ} \mathrm{C}$ for $1 \mathrm{~s}, 65^{\circ} \mathrm{C}$ for $1 \mathrm{~s}$ \\
\hline & Reverse; TGCTGGTAGAGTTCGGTGCAGAAT & Melt curve; $65-95^{\circ} \mathrm{C}$ for $2 \mathrm{sec} / \mathrm{step}$ \\
\hline \multirow[t]{2}{*}{ IFN- $\beta$} & Forward; TGGGAGGCTTGAATACTGCCTCAA & $98^{\circ} \mathrm{C}$ for $30 \mathrm{~s}, 98^{\circ} \mathrm{C}$ for $1 \mathrm{~s}, 65^{\circ} \mathrm{C}$ for $1 \mathrm{~s}$ \\
\hline & Reverse; TCTCATAGATGGTCAATGCGGCGT & Melt curve; $65-95^{\circ} \mathrm{C}$ for $2 \mathrm{sec} /$ step \\
\hline \multirow[t]{2}{*}{ c-fos } & Forward; GCAAGGTGGAACAGTTATCTCCA & $98^{\circ} \mathrm{C}$ for $30 \mathrm{~s}, 98^{\circ} \mathrm{C}$ for $1 \mathrm{~s}, 65^{\circ} \mathrm{C}$ for $1 \mathrm{~s}$ \\
\hline & Reverse; TTCAGAGGTTGGCAATCTGC & Melt curve; $65-95^{\circ} \mathrm{C}$ for $2 \mathrm{sec} / \mathrm{step}$ \\
\hline \multirow[t]{2}{*}{ STAT3 } & Forward; CTGGCCTTTGGTGTTGAAAT & $98^{\circ} \mathrm{C}$ for $30 \mathrm{~s}, 98^{\circ} \mathrm{C}$ for $1 \mathrm{~s}, 65^{\circ} \mathrm{C}$ for $1 \mathrm{~s}$ \\
\hline & Reverse; AAGGCACCCACAGAAACAAC & Melt curve; $65-95^{\circ} \mathrm{C}$ for $2 \mathrm{sec} / \mathrm{step}$ \\
\hline
\end{tabular}


CA, USA). Real-time PCR was performed using the SsoAdvanced ${ }^{\mathrm{TM}}$ SYBR ${ }^{\circledR}$ Green Supermix (Bio-Rad, Hercules, CA, USA) to detect mRNA levels of the E6 genes, PDCD4, STAT3, c-fos and IRF genes. Actin was used as an internal control. Primers of mRNA for real time PCR analysis are listed in Table 1. MicroRNA reverse transcription reactions and microRNA quantitative polymerase chain reactions were performed to detect miR21 using TaqMan ${ }^{\circledR}$ MicroRNA Reverse Transcription Kit and TaqMan ${ }^{\circledR}$ MicroRNA Assays (Applied Biosystems, Foster City, CA, USA), respectively and RNU6B was used as an internal control. The real-time PCR was done in triplicates. Relative expression of mRNA was evaluated and normalized with the expression of actin using the $2-{ }^{\Delta \Delta \mathrm{Ct}}$ method. Relative fold-change was compared with the control condition. Relative expression of miRNA was evaluated in the same way but normalized with the expression of U6.

\section{Statistical analysis}

Data were analyzed with ANOVA, followed by Tukey's multiple comparison tests using Prism 5 (GraphPad software, La Jolla, CA). Differences between experimental groups were considered to be significant at $\mathrm{p}<0.05$.

\section{Results}

Characterization of the constructed vectors and E6 genes expression

The constructed plasmids, pcDNA3.2-16E6D25E and pcDNA3.2-16E6Pro were confirmed with two restriction enzymes, Not I and Apa I. Specific fragments of E6D25E and E6Pro genes in these plasmids were sequenced and the amino acid differences between these genes are shown in Figure 1A. pcDNA3.2-16E6D25E or pcDNA3.2-16E6Pro

\section{A}

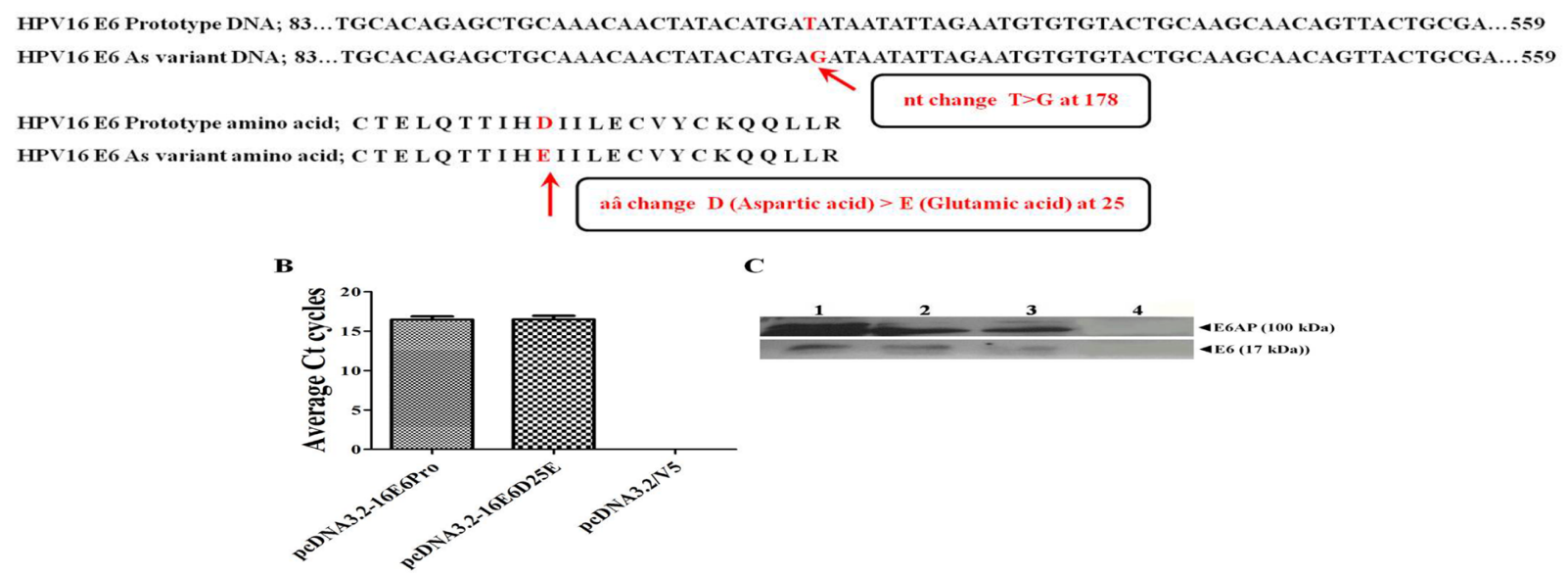

Figure 1. (A) Nucleotide and Amino Acid Differences in the E6 Gene of HPV16As Variants. The HPV16As variant possesses a $\mathrm{G}$ at position 178 (instead of T), leading to an amino acid change from aspartic acid, D, to glutamic acid, E, at position 25. (nt; nucleotide, aâ; amino acid). (B) Amount of E6 mRNA expressed in C33A cells transfected with pcDNA3.2-16E6Pro, pcDNA3.2-16E6D25E and pcDNA3.2/V5 was evaluated using quantitative real-time polymerase chain reaction in triplicate reactions. Values represent mean $\mathrm{Ct}$ cycles $\pm \mathrm{SD}$. Three independent experiments were performed. (C) Immunoprecipitation assay; lysates of co-transfected C33A cells were immunoprecipitated with anti-HPV16/18 E6 antibody. E6AP and HPV16 E6 protein are indicated by arrows. Lane 1 shows results from $\mathrm{SiHa}$ cell lysate, Lane 2 shows results from C33A cells transfected with pcDNA3.2-16E6Pro lysate, Lane 3 shows C33A cells transfected with pcDNA3.2-16E6D25E lysate and Lane 4 shows results from C33A cell lysate

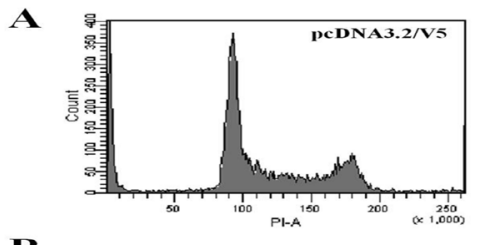

$\mathbf{B}$

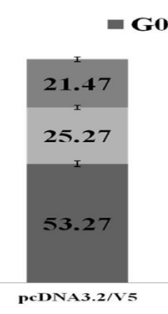

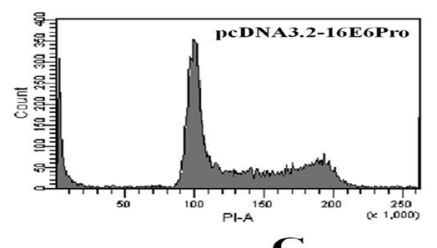

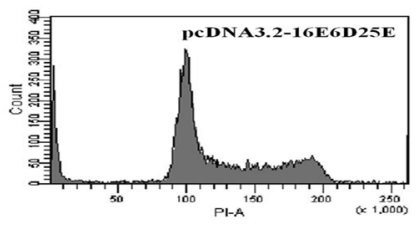

C

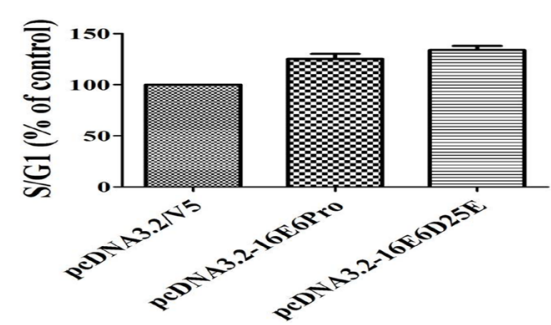

Figure 2. The Cell-cycle Progression by pE6Pro and pE6D25E. C33A cells were transfected with pcDNA3.2-16E6Pro or pE6 pcDNA3.2-16E6D25E or pcDNA3.2/V5 (mock). The distribution of cells in G0/G1, S and G2/M phases was determined in triplicate using flow cytometry. (A) Cell distributions from a representative experiment. (B) Mean percentages of cells in different stages of the cell cycle. (C) Differences in the S/G1 cell-cycle ratio. Mean values \pm SD of at least three independent experiments are presented 
was verified by transient transfection into a C33A cell line, and the result showed that levels of E6 mRNA expression from pcDNA3.2-16E6D25E and pcDNA3.2-16E6Pro were similar (Figure 1B).

\section{Characterization of E6E25D protein functions}

Interaction between E6D25E protein and E6AP was determined and compared to E6Pro protein used as control. pcDNA3.2-16E6D25E or pcDNA3.2-16E6Pro was co-transfected with pcDNA3-E6AP and the protein interaction was determined using an immunoprecipitation method. The result suggested that E6D25E protein still maintained the E6-specific functions and that it could bind to E6AP (Figure 1C). In addition, E6D25E and E6Pro proteins also showed the ability to override cell growth arrest, as determined in transfected C33A cells (Figure 2A) using flow cytometry. There was an increase in the percentage of E6-expressing cells at the $S$ phase entry stage (Figure 2B), as evidenced by an increase in the $\mathrm{S} /$ G1 ratio (Figure 2C). These functions were no different between the E6Pro and E6D25E proteins. Therefore, these vectors were used for further investigations.

\section{Effect of E6D25E on miR-21 expression}

Over-expression of miR-21 has been found in various cancers including cervical cancer. This study demonstrated that E6 protein up-regulated miR-21 expression in C33A cells transfected with pcDNA3.2-16E6D25E and pcDNA3.2-16E6Pro. Interestingly, the variant proteins differed in the extent of this effect. Compared with empty vector control, miR-21 was up-regulated to a greater extent by E6D25E (2.3-fold) than E6Pro (1.6-fold). This is the first report that up-regulation of miR-21 is partly associated with oncoprotein E6 of HPV16 in cervical cancer cells.

MiR-21 expression in HCK1T cells expressing E6Pro or E6D25E protein was very low and not significantly difference from the HCK1T cell control (Figure 3B). This result was confirmed by the level of STAT3 gene expression (the transcriptional activator of miR-21) (Figure 3C). After treatment with HeLa-CM for $48 \mathrm{~h}$, miR-21 expression was induced in these cells. Figure $3 \mathrm{D}$ shows that miR-21 levels increased in HCK1T cells expressing E6Pro and E6D25E in CM treatment, but there was no significant difference between these. Interestingly, when treated with HeLa-CM, up-regulation of miR-21 was significantly greater in HCK1T cells expressing E6D25E than in HCK1T cells expressing E6Pro (Figure 3E). These results demonstrated that E6D25E differs from E6Pro in its ability to regulate miR-21 expression in HCK1T cells under exposure to substances secreted from HPVinfected cells. To confirm the up-regulation of miR-21 in HCK1T cells expressing E6, total RNA was extracted and investigated for PDCD4 expression. The results showed down-regulation of the PDCD4 gene to a greater extent in HCK1T cells expressing E6 proteins and treated with HeLa-CM than with CM (Figure 3F).

\section{Effect of E6D25E on human immune response genes}

To investigate the role of E6D25E in immune surveillance, the expression of IRFs $(1,3,7)$, IFN type I (IFN- $\alpha$ and IFN- $\beta$ ) and c-fos were investigated in the HCK1T cells expressing E6D25E or E6Pro with or without treatment of HeLa-CM. The results showed that, in conditions without treatment, expression of IRF 1, 7 and c-fos genes were significantly down-regulated in HCK1T cells expressing E6D25E or E6Pro as shown in Figure 4A, 4C and 4D. The expression of IRF 3 was significantly suppressed in HCK1T cells expressing E6Pro, but only slightly (and non-significantly) down-regulated in HCK1T cells expressing E6D25E (Figure 4B).

When treated with HeLa-CM, the expression levels of IRF 1 (Figure 4G), 3 (Figure 4H), 7 (Figure 4I) and c-fos (Figure 4J) were significantly suppressed in the HCK1T
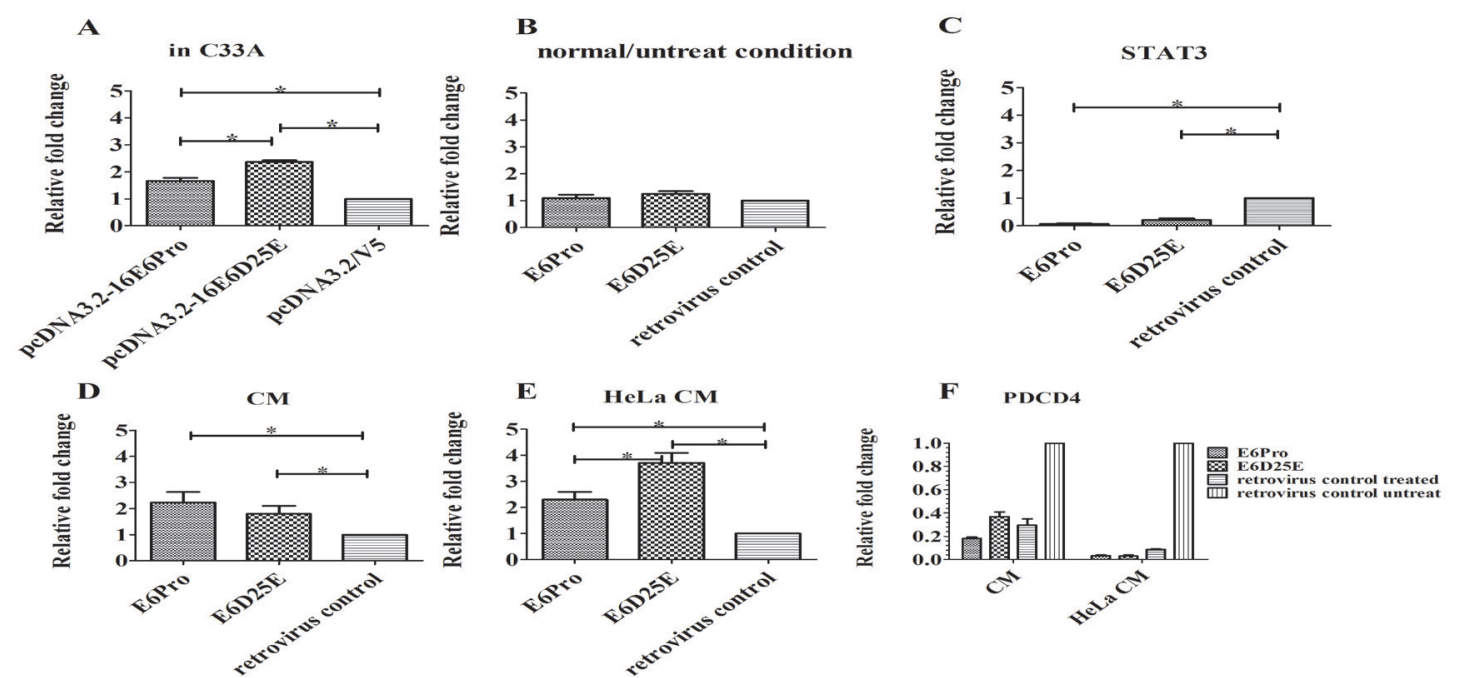

Figure 3. The Expression Levels of miR-21, STAT3 and PDCD4. All experiments were measured in triplicate using real-time PCR. MiR-21 levels in C33A cells transfected with pcDNA3.2-16E6Pro, pcDNA3.2-16E6D25E and pcDNA3.2/V5; A). MiR-21 expression level in HCK1T cells expressing E6D25E or E6Pro and HCK1T cells transduced with retrovirus control under untreated conditions; B). HCK1T cells treated with CM, 10\% FBS in DMEM; D), HCK1T cells treated with HeLa-CM; E). STAT3 mRNA level in HCK1T cells expressing E6D25E or E6Pro and HCK1T cells transduced with retrovirus control under untreated conditions; C). PDCD4 mRNA level in HCK1T cells expressing E6D25E or E6Pro and HCK1T cells transduced with retrovirus control and treated with CM or HeLa-CM; F) 

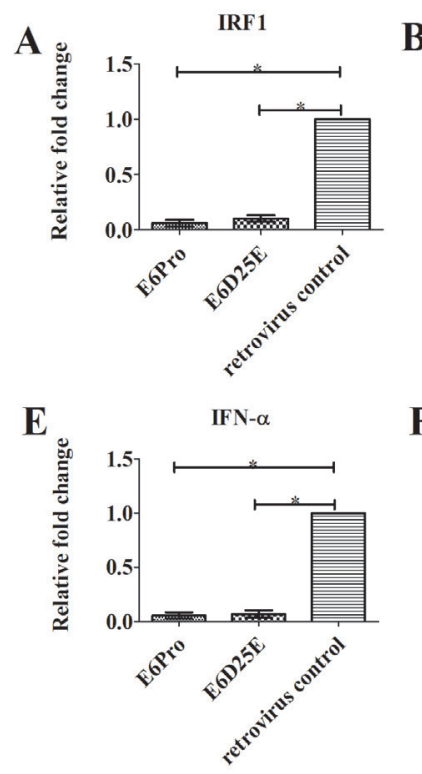

I

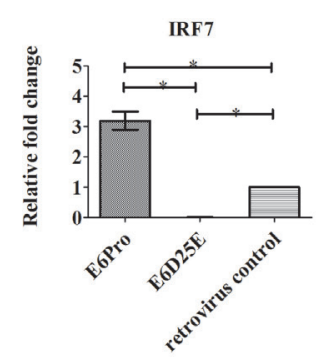

B

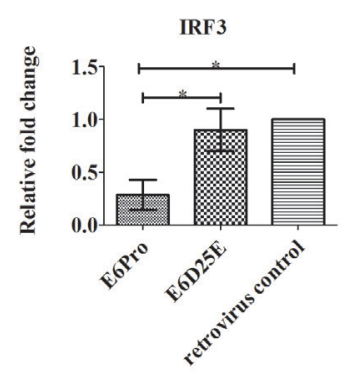

F

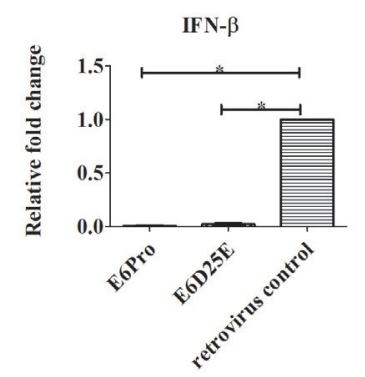

$\mathbf{J}$

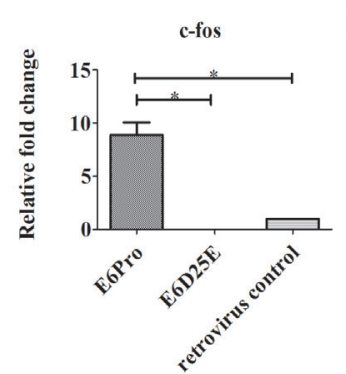

C

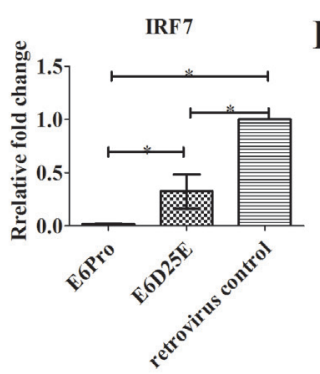

G

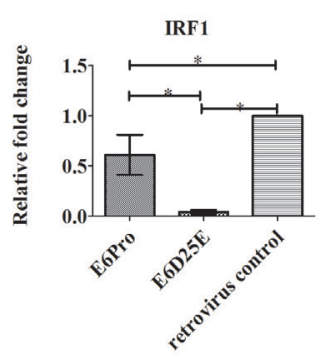

$\mathbf{K}$

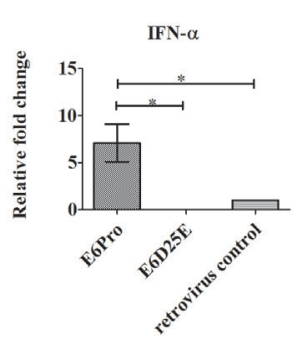

D

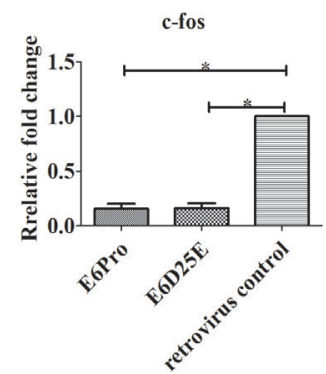

H

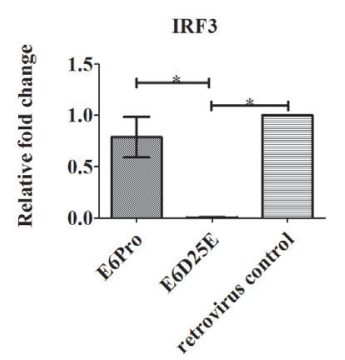

$\mathbf{L}$



Figure 4. The Expression Levels of IRFs, c-fos, IFN- $\alpha$ and IFN- $\beta$. All experiments were done in triplicate using real-time PCR. IRF 1, IRF 3, IRF 7, c-fos, IFN- $\alpha$ and IFN- $\beta$ expression levels in HCK1T cells expressing E6D25E or E6Pro and HCK1T cells transduced with retrovirus control under untreated conditions (A-F). IRF 1, IRF 3, IRF 7, c-foc, IFN- $\alpha$ and IFN- $\beta$ expression levels in HCK1T cells expressing E6D25E or E6Pro and HCK1T cells transduced with retrovirus control and treated with HeLa-CM (G-L)

cells expressing E6D25E, but suppressed to a far lesser extent in the HCK1T cells expressing E6Pro. Interestingly, the downstream signaling genes, IFN- $\alpha$ and IFN- $\beta$, were significantly suppressed in HCK1T cells expressing E6D25E or E6Pro even when not treated with HeLa-CM (Figure 4E -4F). However, when treated with HeLa-CM, IFN- $\alpha$ and IFN- $\beta$ were significantly up-regulated in the HCK1T cells expressing E6Pro (Figure 4K -4L). This result suggested that E6D25E differs from E6Pro in its ability to inhibit expression of IFN type I and differentially affects the ability of HCK1T cells to respond to mediators in HeLa-CM.

\section{Discussion}

This study compared the activities of E6D25E protein with those of E6Pro protein. Vectors expressing E6Pro and E6D25E were constructed and the function of E6 proteins, including binding to E6AP and overriding cell growth arrest, was confirmed using immunoprecipitation and flow cytometry, respectively. The relative levels of miR-21 in C33A cells expressing E6D25E or E6Pro, and in HCK1T expressing E6D25E or E6Pro, were investigated using real-time PCR. The results demonstrated that E6D25E likely has oncogenicity similar to that of E6Pro, but showed a higher ability to induce miR-21 expression. Expression of IRF 1, 3 and 7 genes and c-fos gene also differed between HCK1T cells expressing E6D25E and those expressing E6Pro, with or without HeLa-CM treatment.

E6D25E is similar to E6Pro in its ability to bind to E6AP. This ability is commonly found in HR-HPV infected cervical cells and effects p53 inactivation and degradation via the ubiquitination pathway. This subsequently causes loss of regulation of cell-cycle progression (Tungteakkhun and Duerksen-Hughes, 2008) and contributes to accumulation of genetic alteration involved in cervical cancer progression (Howie et al., 2009). The ability of E6 to degrade p53 was observed in our previous study: E6D25E and E6Pro show similar abilities to decrease p53 and p21 levels in HCK1T cells expressing E6D25E or E6Pro, corresponding with the low expression levels of p21 CIP/WAF1 (Yi et al., 2013; Hang et al., 2014). Abolition of p53 induces cell entry into the S-phase and completion of DNA synthesis, a condition that causes DNA damage (Taylor and Stark, 2001). An ability of E6D25E to stimulate the cell cycle into the $S$ phase was also detected in C33A cells expressing E6D25E or E6Pro.

Up-regulation of miR-21 has been detected both in cervical cell lines (Wang et al., 2008) and in cervical cancer tissues (Lui et al., 2007). However, some studies did not find this to be the case in cervical cancer tissues (Martinez et al., 2008; Li et al., 2011; Rao et al., 2012). Thus, the association of HR-HPV and miR-21 upregulation with cervical cancer development is still limited. Our study demonstrated that the HPV16 E6 
proteins induced up-regulation of miR-21. Interestingly, E6D25E caused greater levels of induction than did E6Pro, suggesting that the former may have a greater influence on cell immune resistance and survival (Young et al., 2010). In addition, this study demonstrated that induction of miR-21 might be dependent on microenvironment mediators affecting activity of E6Pro and E6D25E. For example, miR-21 is significantly up-regulated in HCK1T cells expressing E6D25E or E6Pro and incubated with HeLa-CM. This result suggestes that the up-regulation of miR-21 is found not only in cancer cells but also a common feature in cell stress. Yao at el (2011) showed that miR-21 expression was very sensitive to CM stimulation as well as to TGF- $\beta$ that is commonly found in HeLa-CM (Yao et al., 2011). Overexpression of STAT3 has also been reported in cervical cancer (Sobti et al., 2009; Shukla et al., 2010). Strong evidence has been found that HPV 16/18 E6 protein can activate STAT3 to enhance secretion of IL-6 into the cell matrix (Ren et al., 2013), and STAT3 was reported as an enhancer element in the promoter of miR-21 (Li et al., 2012). These data may support the different ability of E6D25E from E6Pro to activate STAT3 expression or response to mediators. Corresponding with these studies, up-regulation of miR-21 was significantly greater in HCK1T cells expressing E6D25E than in those expressing E6 Pro when treated with HeLa-CM. This result was confirmed by reduction of PDCD4, a direct target of miR-21 in the HCK1T cells expressing E6D25E or E6Pro (Figure 3F). The functions of PDCD4 involve apoptosis, blocking of translation and inhibition of tumor growth (Yao et al., 2009). Previous reports showed that high expression of miR-21 leads to decreased PDCD4 expression, increased expression of interleukin (IL)-10 and decreased NFxB activity (Sheedy et al., 2010).

Previous evidence has shown that HR-HPV E7 interacts with IRF 1 and inactivates its transactivation function. Therefore, it could be speculated that HR-HPV E6 and E7 oncoproteins interfere with IFN signaling, leading to viral evasion from host immunity in cervical disease (Park et al., 2000). This study showed suppression of IRF 1 in the HCK1T cells expressing E6D25E and E6Pro. However, different expression patterns of IRF 3 and 7 were observed. These are the key regulators of IFN type I gene expression upon virus infection. Corresponding with this study, E6D25E and E6Pro also affected on IFN Type I, IFN- $\alpha$ and IFN- $\beta$ expression in the HCK1T cells expressing E6D25E or E6Pro. IFN Type I are activated downstream of certain PRR including TLR3, TLR4, TLR7, TLR8, TLR9, RIG-I, MDA5 and DAI (DLM-1/ZBP1), and undergo nuclear translocation for type IFN I induction (Takaoka et al., 2008). A previous study reported that EBV latent membrane protein 1 (LMP-1) regulated expression and activation of IRF 7 via phosphorylation (Zhang and Pagano, 1997). Our result suggests the differing activities of E6D25E on protein interactions, with and without the presence of mediators such as in HeLa-CM, may enhance the oncogenic role of miR-21 in cells infected with HPV16As. This study demonstrated that IRF 1, 3 and 7, as well as c-fos, were significantly suppressed in HCK1T cells expressing E6D25E and treated with HeLa-CM, whereas in HCK1T cells expressing E6Pro, these molecules were induced. This result may suggest a different ability of E6D25E to evade immune surveillance from E6Pro.

In conclusion, E6D25E of the HPV16As variant differed from the E6 prototype in its activities on modulation of miR-21 expression and expression of host immune genes. These activities may contribute persistent infection that might be a key factor for the important role of this variant in cervical cancer progression.

\section{Acknowledgements}

We are grateful to Prof. Tohro Kiyono (National Cancer Center Research Institute, Japan) for supporting throughout this work. This study was granted by Faculty of Medicine, Khon Kaen University, Thailand (Grant Number I55112) and Research Grant for Mid-Career University Faculty, RMU (Grant Number RMU5380038). We would like to acknowledge Prof. David Blair for editing the MS via Publication Clinic KKU, Thailand.

\section{References}

Barnard P, McMillan NA (1999). The human papillomavirus E7 oncoprotein abrogates signaling mediated by interferonalpha. Virology, 259, 305-13.

Chang YJ, Chen HC, Pan MH, et al (2013). Intratypic variants of human papillomavirus type 16 and risk of cervical neoplasia in Taiwan. J Med Virol, 85, 1567-76.

Choi BS, Kim SS, Yun H, et al (2007). Distinctive distribution of HPV16 E6 D25E and E7 N29S intratypic Asian variants in Korean commercial sex workers. J Med Virol, 79, 426-30.

Chopjitt P, Ekalaksananan T, Pientong C, et al (2009). Prevalence of human papillomavirus type 16 and its variants in abnormal squamous cervical cells in Northeast Thailand. Int J Infect Dis, 13, 212-9.

Cornet I, Gheit T, Franceschi S, et al (2012). Human papillomavirus type 16 genetic variants: phylogeny and classification based on E6 and LCR. J Virol, 86, 6855-61.

Gheit T, Cornet I, Clifford GM, et al (2011). Risks for persistence and progression by human papillomavirus type 16 variant lineages among a population-based sample of Danish women. Cancer Epidemiol Biomarkers Prev, 20, 1315-21.

Hang D, Gao L, Sun M, et al (2014). Functional effects of sequence variations in the E6 and E2 genes of human papillomavirus 16 European and Asian variants. $J$ Med Virol, 86, 618-26.

Howie HL, Katzenellenbogen RA, Galloway DA (2009). Papillomavirus E6 proteins. Virology, 384, 324-34.

Huibregtse JM, Scheffner M, Howley PM (1993). Localization of the E6-AP regions that direct human papillomavirus E6 binding, association with $\mathrm{p} 53$, and ubiquitination of associated proteins. Mol Cell Biol, 13, 4918-27.

Kang S, Jeon YT, Kim JW, et al (2005). Polymorphism in the E6 gene of human papillomavirus type 16 in the cervical tissues of Korean women. Int J Gynecol Cancer, 15, 107-12.

Kavitha N, Vijayarathna S, Jothy SL, et al (2014). MicroRNAs: biogenesis, roles for carcinogenesis and as potential biomarkers for cancer diagnosis and prognosis. Asian Pac J Cancer Prev, 15, 7489-97.

Kiyono T, Hiraiwa A, Fujita M, et al (1997). Binding of highrisk human papillomavirus E6 oncoproteins to the human homologue of the Drosophila discs large tumor suppressor protein. Proc Natl Acad Sci USA, 94, 11612-6.

Li S, Liang Z, Xu L, et al (2012). MicroRNA-21: a ubiquitously 
expressed pro-survival factor in cancer and other diseases. Mol Cell Biochem, 360, 147-58.

Li Y, Liu J, Yuan C, et al (2010). High-risk human papillomavirus reduces the expression of microRNA-218 in women with cervical intraepithelial neoplasia. J Int Med Res, 38, 1730-6.

Li Y, Wang F, Xu J, et al (2011). Progressive miRNA expression profiles in cervical carcinogenesis and identification of HPV-related target genes for miR-29.J Pathol, 224, 484-95.

Lui WO, Pourmand N, Patterson BK, et al (2007). Patterns of known and novel small RNAs in human cervical cancer. Cancer Res, 67, 6031-43.

Martinez I, Gardiner AS, Board KF, et al (2008). Human papillomavirus type 16 reduces the expression of microRNA-218 in cervical carcinoma cells. Oncogene, 27, 2575-82.

Mosmann JP, Monetti MS, Frutos MC, et al (2015). Mutation detection of E6 and LCR genes from HPV 16 associated with carcinogenesis. Asian Pac J Cancer Prev, 16, 1151-7.

Narisawa-Saito M, Yoshimatsu Y, Ohno S, et al (2008). An in vitro multistep carcinogenesis model for human cervical cancer. Cancer Res, 68, 5699-705.

Park JS, Kim EJ, Kwon HJ, et al (2000). Inactivation of interferon regulatory factor- 1 tumor suppressor protein by HPV E7 oncoprotein. Implication for the E7-mediated immune evasion mechanism in cervical carcinogenesis. $J$ Biol Chem, 275, 6764-9.

Pientong C, Wongwarissara P, Ekalaksananan T, et al (2013). Association of human papillomavirus type 16 long control region mutation and cervical cancer. Virol J, 10, 30.

Rao Q, Shen Q, Zhou H, et al (2012). Aberrant microRNA expression in human cervical carcinomas. Med Oncol, 29, 1242-8.

Ren C, Cheng X, Lu B, et al (2013). Activation of interleukin-6/ signal transducer and activator of transcription 3 by human papillomavirus early proteins 6 induces fibroblast senescence to promote cervical tumourigenesis through autocrine and paracrine pathways in tumour microenvironment. Eur $J$ Cancer, 49, 3889-99.

Ronco LV, Karpova AY, Vidal M, et al (1998). Human papillomavirus 16 E6 oncoprotein binds to interferon regulatory factor-3 and inhibits its transcriptional activity. Genes Dev, 12, 2061-72.

Sasagawa T, Takagi H, Makinoda S (2012). Immune responses against human papillomavirus (HPV) infection and evasion of host defense in cervical cancer. J Infect Chemother, $\mathbf{1 8}$, 807-15.

Schiffman M, Rodriguez AC, Chen Z, et al (2010). A population-based prospective study of carcinogenic human papillomavirus variant lineages, viral persistence, and cervical neoplasia. Cancer Res, 70, 3159-69.

Sheedy FJ, Palsson-McDermott E, Hennessy EJ, et al (2010). Negative regulation of TLR4 via targeting of the proinflammatory tumor suppressor PDCD4 by the microRNA miR-21. Nat Immunol, 11, 141-7.

Shukla S, Shishodia G, Mahata S, et al (2010). Aberrant expression and constitutive activation of STAT3 in cervical carcinogenesis: implications in high-risk human papillomavirus infection. Mol Cancer, 9, 282.

Smith EJ, Marie I, Prakash A, et al (2001). IRF3 and IRF7 phosphorylation in virus-infected cells does not require double-stranded RNA-dependent protein kinase R or Ikappa B kinase but is blocked by Vaccinia virus E3L protein. J Biol Chem, 276, 8951-7.

Sobti RC, Singh N, Hussain S, et al (2009). Overexpression of STAT3 in HPV-mediated cervical cancer in a north Indian population. Mol Cell Biochem, 330, 193-9.

Suthipintawong C, Siriaunkgul S, Tungsinmunkong K, et al (2011). Human papilloma virus prevalence, genotype distribution, and pattern of infection in Thai women. Asian Pac J Cancer Prev, 12, 853-6.

Takaoka A, Tamura T, Taniguchi T (2008). Interferon regulatory factor family of transcription factors and regulation of oncogenesis. Cancer Sci, 99, 467-78.

Taylor WR, Stark GR (2001). Regulation of the G2/M transition by p53. Oncogene, 20, 1803-15.

Tungteakkhun SS, Duerksen-Hughes PJ (2008). Cellular binding partners of the human papillomavirus E6 protein. Arch Virol, 153, 397-408

Um SJ, Rhyu JW, Kim EJ, et al (2002). Abrogation of IRF-1 response by high-risk HPV $\mathrm{E} 7$ protein in vivo. Cancer Lett, 179, 205-12.

Walboomers JM, Jacobs MV, Manos MM, et al (1999). Human papillomavirus is a necessary cause of invasive cervical cancer worldwide. J Pathol, 189, 12-9.

Wang X, Tang S, Le SY, et al (2008). Aberrant expression of oncogenic and tumor-suppressive microRNAs in cervical cancer is required for cancer cell growth. PLoS One, $\mathbf{3}, 2557$.

Xi LF, Koutsky LA, Hildesheim A, et al (2007). Risk for highgrade cervical intraepithelial neoplasia associated with variants of human papillomavirus types 16 and 18. Cancer Epidemiol Biomarkers Prev, 16, 4-10.

Xie H, Zhao Y, Caramuta S, et al (2012). miR-205 expression promotes cell proliferation and migration of human cervical cancer cells. PLoS One , 7, 46990.

$\mathrm{Xu}$ YZ, Xi QH, Ge WL, et al (2013). Identification of serum microRNA-2 1 as a biomarker for early detection and prognosis in human epithelial ovarian cancer. Asian Pac J Cancer Prev, 14, 1057-60.

Yablonska S, Hoskins EE, Wells SI, et al (2013). Identification of miRNAs dysregulated in human foreskin keratinocytes (HFKs) expressing the human papillomavirus (HPV) Type 16 E6 and E7 oncoproteins. Microrna, 2, 2-13.

Yamada T, Manos MM,Peto J, et al (1997). Human papillomavirus type 16 sequence variation in cervical cancers: a worldwide perspective. J Virol, 71, 2463-72.

Yao Q, Cao S, Li C, et al (2011). Micro-RNA-21 regulates TGF-beta-induced myofibroblast differentiation by targeting PDCD4 in tumor-stroma interaction. Int J Cancer, 128, 1783-92.

Yao Q, Xu H, Zhang QQ, et al (2009). MicroRNA-21 promotes cell proliferation and down-regulates the expression of programmed cell death 4 (PDCD4) in HeLa cervical carcinoma cells. Biochem Biophys Res Commun, 388, 539-42.

Yi JW, Jang M, Kim SJ, et al (2013). Degradation of p53 by natural variants of the E6 protein of human papillomavirus type 16. Oncol Rep, 29, 1617-22.

Young MR, Santhanam AN, Yoshikawa N, et al (2010). Have tumor suppressor PDCD4 and its counteragent oncogenic miR-21 gone rogue? Mol Interv, 10, 76-9.

Zehbe I, Wilander E, Delius H, et al (1998). Human papillomavirus 16 E6 variants are more prevalent in invasive cervical carcinoma than the prototype. Cancer Res, 58, 829-33.

Zhang EY, Tang XD (2012). Human papillomavirus type 16/18 oncoproteins: potential therapeutic targets in non-smoking associated lung cancer. Asian Pac J Cancer Prev, 13, 5363-9.

Zhang L, Pagano JS (1997). IRF-7, a new interferon regulatory factor associated with Epstein-Barr virus latency. Mol Cell Biol, 17, 5748-57. 